\title{
Testing AND Deployment OF INTELLIGENT OBJECT FRAMEWORK
}

\author{
Sasa Savicand Hao Shi \\ College of Engineering and Science, Victoria University, Melbourne, Australia
}

\begin{abstract}
Intelligent Object Framework (IOF) is a platform independent framework allowing different devices to be controlled, monitored and communicated. The Core Framework is developed using Microsoft Visual Studio, Microsoft's .NET Framework and Microsoft's Windows Mobile SDK. Secondary Intelligent Object is developed using Tibbo Integrated Development Environment (TIDE) and T-BASIC programming language that is loaded on an EM1026 Embedded Device Platform running Tibbo Operating System (TiOS). The backend is using Microsoft's SQL Server.The successful implementation of the IOF reliesheavily on the testing and deployment which allows seamless monitoring and control of two intelligent objects without any communication glitch between HTC Windows Mobile enabled device and Tibbo EM1026 embedded module. In this paper, embedded systems and Bluetooth applications are first reviewed. The integration testing including First Time Access, Function Exchange, and Function Execution is presented and then followed by deployment of the IOF and the associated components. Finally conclusions are drawn.
\end{abstract}

\section{KEYWORDS}

Intelligent Object Framework, Embedded Systems, Bluetooth and Wireless Network.

\section{INTRODUCTION}

"A mass in movement resists change of direction. So does the world oppose a new idea? It takes time to make up the minds to its value and importance. Ignorance, prejudice and inertia of the old retard its early progress. It is discredited by insincere exponents and selfish exploiters. It is attacked and condemned by its enemies. Eventually, though, all barriers are thrown down, and it spreads like fire. This will also prove true of the wireless art.'[1]. Those were the words spoken over one hundred years ago by Nikola Tesla, the father of modern day Alternating Current (AC) and the first person to patent radio technology [2].

Human society has come a long way since then and is continuing to spiral towards the never ending technological advancements. With the introduction of the Internet in 1990's [3] to the general public, the world of communication has spread its wings far and wide to the pinnacles of the world creating a fast spreading web of information and data. To accompany the growing data exchange and communication frenzy, there has been exponential growth in communication devices, data transfer standards and software technologies and methodologies. Each one contributing significantly to the other in order to work in perfect harmony in creating some of the world's most marvellous communication mediums such as the very internet we use every day. Email communications, data storage and retrieval, device control and monitoring, remote assistance and many more. The sophisticated hardware devices that managed data transfers would not be what they are without the underlying programs which ran them deep within their core. DOI : 10.5121/ijsea.2013.4601 
Taking things even further into lower levels of their infrastructure, Embedded Systems play an equally important role.

In this paper, the core embedded technologies and their underlying management processes are reviewed. Current development environments of the IOF that helped in shaping managed applications facilitate developers and systems designers come up with new innovative, fast and reliable methodologies and applications that are being used [4, 5]. The integration testing including First Time Access, Function Exchange, and Function Execution are covered. The deployment of the IOF and the associated components are presented with a hopeful endeavour on how to utilize the functionality to accomplish some basic control structures via a protocol standard through a wireless medium [6].

\section{EMBEDDED SYSTEMS}

The earliest embedded systems were making use of microprocessor development specifically for those kinds of applications and they were typically known as microcontrollers. Each new product required a costly and labour-intensive development phase [7]. One of the most surprising developments of the last few decades has been the ascendance of computers in our homes, yet many of these computers and their counterpart smaller components and systems are not recognized as such by their users. The first such system was introduced by Intel, the world's first single chip microprocessor in 1971 [8]. Figure 1 shows the chip's physical layout and the internal structure.

An embedded system is a combination of hardware and software components which are designed to carry out dedicated functions in order to achieve an output of some sort. A good example of a device housing an embedded system would be a Wi-Fi router or a mobile phone. Effectively, a device such as a mobile phone or a Wi-Fi router has a number of embedded modules, each one programmed specifically to handle the type of input/output designated. One embedded module might be specific to a keypad, whilst another is responsible for the LCD display of the mobile phone. Wi-Fi router wouldn't have many embedded units within the structure for the reason that it is very specific in its set of operations. Modulated signals are sent and received over a wireless medium with a couple of helper processors carryingthe encoding and the decoding process of the data.
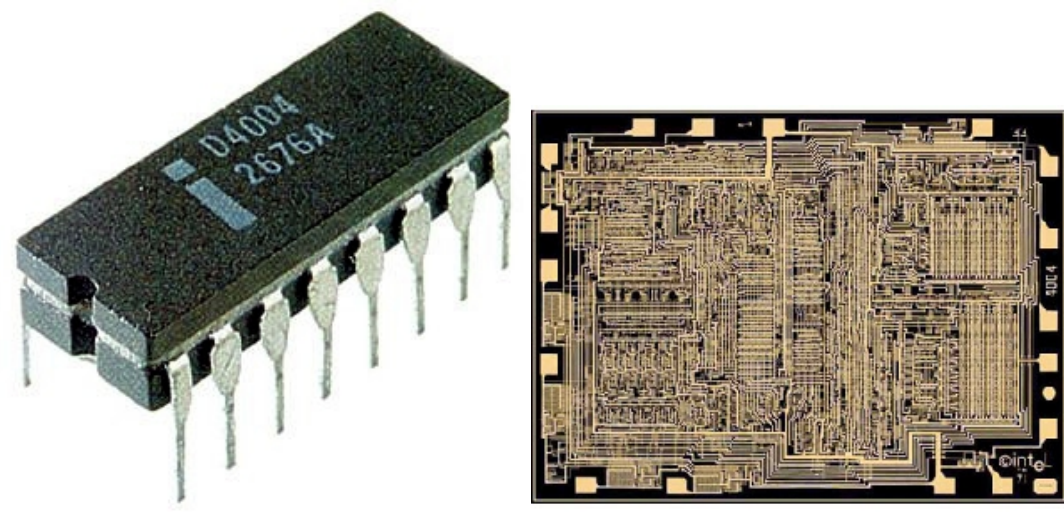

Figure 1. Intel 4004 Microprocessor, 1971 [6] 
International Journal of Software Engineering \& Applications (IJSEA), Vol.4, No.6, November 2013

As the years progressed, the designs were becoming more and more complex but utilizing a lot of features within their processors which were getting smaller with each new design implementation. This paved a way of unimaginable possibilities of implementation in the real world providing people with the ability to become their own masters of design. The details of the Embedded Systems Development are summarized in Table 1.

Table 1: Timeline of Embedded Systems Design[11]

\begin{tabular}{|c|c|}
\hline Year & Event \\
\hline $1936-1937$ & $\begin{array}{l}\text { Alan Turin publishes article "On Computable Numbers, with an Application to } \\
\text { Entscheindungsproblem", which paves the way not only for computers but for } \\
\text { stored-program architecture. }\end{array}$ \\
\hline 1939 & $\begin{array}{l}\text { John Atanasoff and Clifford Berry create a prototype of the Atanasoff-Berry } \\
\text { Computer }(\mathrm{ABC}) \text {, the first digital computer. }\end{array}$ \\
\hline 1944 & $\begin{array}{l}\text { Colossus, the first fully functioning electric digital computer, used by the } \\
\text { Bletchley Park cryptanalysis team. }\end{array}$ \\
\hline 1946 & $\begin{array}{l}\text { Eniac, the world's first "programmable" stored program electronic computer } \\
\text { comes to life. It housed 18,000 vacuum tubes and it roughly had the complexity } \\
\text { of today's cheap digital watch. }\end{array}$ \\
\hline 1962 & Texas Instruments introduces the 7400 series of logic IC's \\
\hline 1965 & $\begin{array}{l}\text { DEC introduces the } 12 \text { bit PDP- } 8 \text { minicomputer, which by } 1973 \text { was the best- } \\
\text { selling computer in the world at a staggering cost of } \$ 18 \mathrm{k} \text { in } 1965 \text { dollars, or } \\
\$ 120 \mathrm{k} \text { today }\end{array}$ \\
\hline 1970 & $\begin{array}{l}\text { Intel releases the } 1103 \text { chip, the first generally available dynamic random-access } \\
\text { memory (DRAM) chip. }\end{array}$ \\
\hline 1971 & Intel releases first microprocessor, the 4004, a 4bit central processing unit. \\
\hline 1971 & $\begin{array}{l}\text { Intel releases the } 1101 \text { chip, a } 256 \text { bit programmable memory, and the } 1701 \text { chip, } \\
\text { a } 256 \text { byte erasable read-only memory (EROM) }\end{array}$ \\
\hline 1972 & Intel introduces the 8008 microprocessor \\
\hline 1973 & Intel releases the Intellec 8 development system for the 8008 processor \\
\hline 1973 & $\begin{array}{l}\text { Texas Instruments introduces the first dynamic random-access memory (DRAM) } \\
\text { that is a 4k-bit chip }\end{array}$ \\
\hline 1975 & $\begin{array}{l}\text { MITS releases the ATLAIR } 8800 \text { at an unheard-of price of } \$ 400 \text {. The home } \\
\text { computer was born. }\end{array}$ \\
\hline 1978 & Intel introduces the 8086 microprocessor chip. The beginning of x 86 architecture \\
\hline 1989 & Intel releases the i486, 32-bit x86 microprocessor. \\
\hline 1993 & Intel releases Pentium processor \\
\hline 2002 & Intel Itenium 2 is released \\
\hline 2005 & IBM, Intel and AMD release their first multicore processors \\
\hline
\end{tabular}

Embedded systems have become an essential part of daily lives enabling us to undertake our daily duties in a much simpler and quicker way. Whilst a lot of people accept these technological advancements as a positive drive for human inspiration and exploration, nevertheless it did impact our natural way of thinking. Whilst more and more work is done by automated processes, human involvement in manual labour has drastically decreased. Islam Hassan El-Adaway says "one can trace that as a man reaches more and more progress, he loses more and more of his good instinctive human nature" [11], however according to Jacob Bronowski, "Man is unique not because he does science, and he is unique not because he does art, but because science and art are expressions of his marvellous plasticity of mind". So, in respect to the great minds of the 20th and 21 st century, we continue to evolve our systems based on their legacy, making them more robust and efficient in order to prove ourselves that we are more than capable of taking them to the next level. 
International Journal of Software Engineering \& Applications (IJSEA), Vol.4, No.6, November 2013

Embedded systems have come at such a low cost, that just about anyone with basic electronic and programming knowledge can design an efficient system. Progression of the technology of Embedded Systems has also enabled people to easily scope out, design, build and deploy their prototypes or full scale products ready for the commercial world. The advantage of Embedded Systems is the integration capability with other embedded Devices. This suggests that design may integrate many numbers of other embedded components allowing thedevice

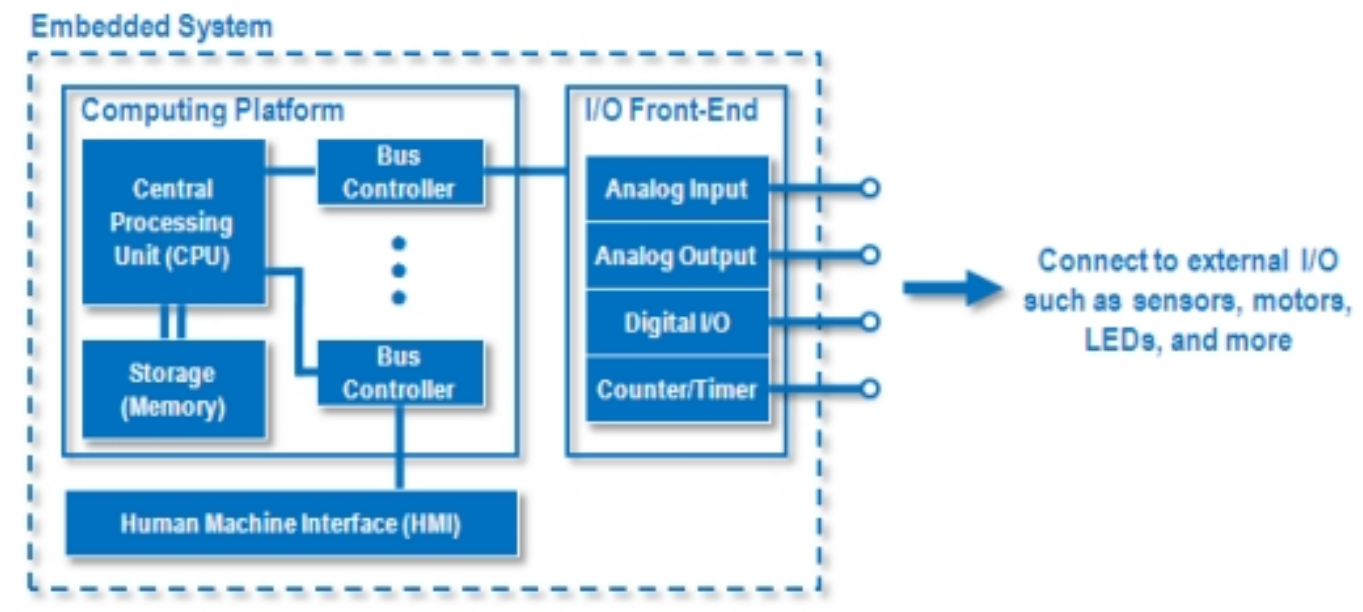

Figure 2. Simple Embedded System [9]

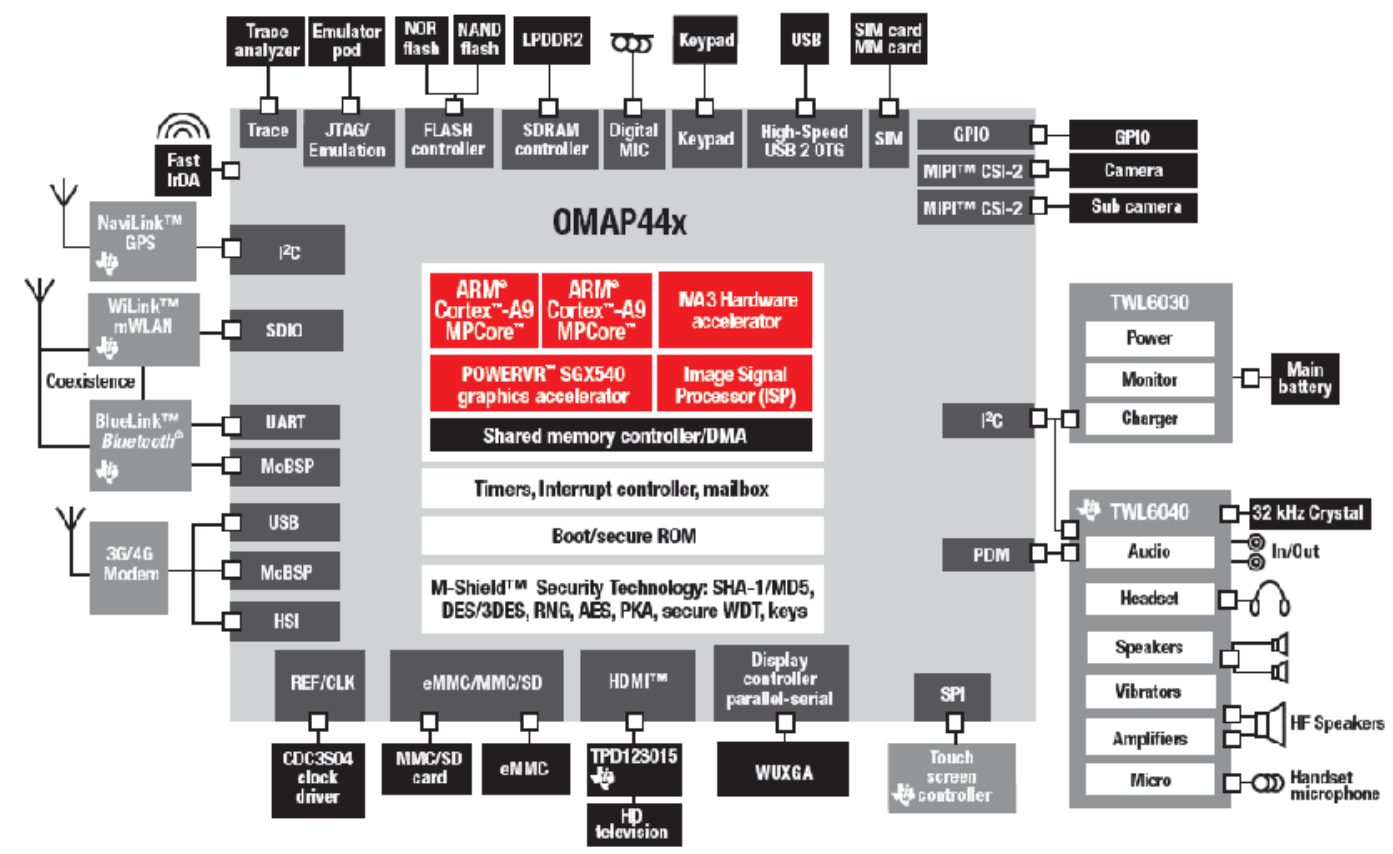

Figure 3. Texas Instruments OMAP Embedded Controller [13]

a wide range of functionalities. Robotics is a perfect blend of integration factors when designing such complex pieces of equipment (this includes the complex industrial robots in addition to the 
International Journal of Software Engineering \& Applications (IJSEA), Vol.4, No.6, November 2013

popular Honda's ASIMO robot). They encapsulate a wide number of different components such as:

\author{
- $\quad$ Motion detection sensors \\ - Ultra-sonic collision detection sensors \\ - Camera controllers \\ - $\quad$ Servo motor controllers - movement of the limbs or other parts \\ - $\quad$ Pressure sensors
}

Each of these components, depending on the nature of their complexity, has an embedded module talking to one another and communicating directly or through a series of other embedded components that act as a central intelligence of communication. The basic layout of an embedded system is shown in Figure 2.A more complex diagram is outlined in Figure 3 where Texas Instruments have introduced a new Open Multimedia Application Platform (OMAP) 4 used for mobile applications. It is to target smart-phones and Mobile Internet Devices (MID) and it introduces a number of benefits over other processors [12]. It features three hardware accelerators to support 1080p, Dual-core ARM Symmetric Multiprocessors and graphics accelerators. With a $1 \mathrm{GHz}+$ processor, this means that a mini super-computer is in a pocket. The sheer complexity of the design can clearly be seen. Essentially the basic components of any embedded systems are the Central Processing Unit (CPU), BUS Controllers, some form of interfaces and an I/O front end which is the bridge to external I/O devices such as the ones mentioned previously.

\title{
3. BLUETOOTH APPLICATIONS
}

Bluetooth RF (physical layer) operates in unlicensed ISM band at $2.4 \mathrm{GHz}$. The symbol rate is 1 mega-symbol per second, supporting bit rates of 1 Megabit per second or with Enhanced Data Rate, a gross air bit rate of 2 or 3Mb/s. There are two modes, Basic Rate and Enhanced Data Rate (EDR). Bluetooth technology has the ability to link digital devices within a range of $10 \mathrm{~m}$, which can be expandable to $100 \mathrm{~m}$ by increasing the transmission power. The most basic overview of Bluetooth can be divided in two areas: Radio and Link \& Channel Management Protocols.

The Radio Channel, during a typical operation, is shared by group of devices that are synchronized to a common clock and frequency hopping pattern. A secondary component of the operation of Bluetooth is the Piconet, and is described as an ad-hoc computer network linking a user group of devices. Bluetooth technology utilizes this network allowing master to slave communication of 7 devices that can be extended to 255. One device provides the synchronization reference and is referred to as the master. The other devices are classified as slaves. Devices in Piconet use a distinctive frequency hopping pattern (FHP) which is algorithmically determined [14].

Link and Channel Management Protocols consist of control layers that sit above the physical channel. The hierarchy consists of channels and links from the physical channel upwards, physical link, logical transport, and logical link and L2CAP channel. Within a physical channel, physical links are formed between two devices that transmit packets in either direction. In a Piconet physical channel, there are restrictions to which devices are allowed to form a physical link. The physical links are only allowed between slave and master, whereas a physical link is not directed between the slaves.

The logical link is used as a transport for one or more logical links that support uni-cast synchronous, asynchronous and isochronous traffic. The link is multiplexed onto the physical 
layer by occupying slots assigned by a scheduling function and for resource management of communication. Link Manager Protocol (LMP) is used to control the operation of devices in the Piconet, thus providing services to manage the lower levels of architectural layers (radio and baseband). Finally above all layers is the L2CAP layer which provides a channel-based abstraction to applications and services.

Bluetooth home based automation systems have been used wildly within homes and cars over the last decade. The proposed system [15] is an adoption of HAP (House Automation Protocol) which works on the Bluetooth communication by establishing a PAN (Personal Area Network) of devices through utilization of Piconet. The system proposes a network which contains a remote, mobile host controller and several client modules, acting as home appliances. The client modules communicate with the host controller through Bluetooth devices. The HAP protocol is constructed above the Bluetooth software stack and it follows the layer model proposed by Bluetooth Special interest Group (SIG). The HAP protocol is described by the home automation descriptor table, which explains the HAP protocol stack. In order to show the proposed system in action, significant software development need to be fulfilled in order to provide the custom firmware for the microcontroller which was driving a room temperature sensor circuitry. The attempt at home automation systems is a successful one but fails to address the issue of complexity in circuitry design and development. The Intelligent Object Framework attempts to hide the complexity from the user by utilization of an adaptive software based protocol stack that can be implemented on a platform that does not need to conform to a specific architectural limitations or design. This can be an ad-hoc network or a specified Wi-Fi, 3G or Ethernet. Limitations with running Piconet PAN's are clearly shown in their lack of direct communication with slave to slave. The multiplexing of data and communication is done via a master controller. The limitations on communication can highly depend on the initial designs of host controllers. Intelligent Object Framework mitigates the limitation by providing a protocol that is scalable across multiple networks and devices. This can further be extended to include device to device communication, as protocol runs on IP.

\section{SYSTEM OVERVIEW}

Intelligent Object Framework is a system of integrated set of services and components which leverage across a scalable network. The complete system provides the user with ability to control devices. A typical scenario of the system is an Intelligent Object enabled device such as an air conditioner. Once the air conditioner is set up, the user discovers IOF Services by initializing a discovery process. Once the protocol is set up and connection established, the IOF Services request device functions. The functions received are stored away in the database, and can be retrieved at any time. This enables quick and easy device access from anywhere in the world provided that the services are visible to the internet. The Intelligent Object Framework specification defines the protocol standard for function exchange. By following the specification, the functionality can be abstracted from the user, and integrated on any platform. The different spectrum of devices that can utilize the framework can range from house hold appliances such as TV's, fridges, air conditioning and heating system to portable device range such as mobile phones and PDA's. The implementation can also be adapted by new breed of Intelligent Cars that are WiFi enabled, with programmable units.

\section{INTEGRATION TESTING}

The framework provides a client application (IOF Management Studio) that uses its services (IOF Service and Protocol Layer) allowing device control and monitoring over Wi-Fi medium $[4,5]$. 
International Journal of Software Engineering \& Applications (IJSEA), Vol.4, No.6, November 2013

The Data Layer allowed fast device function storage and on demand retrieval [6]. The testing is focused on First Time Access, Function Exchange, and Function Execution.

\section{Table 1.First Time Access}

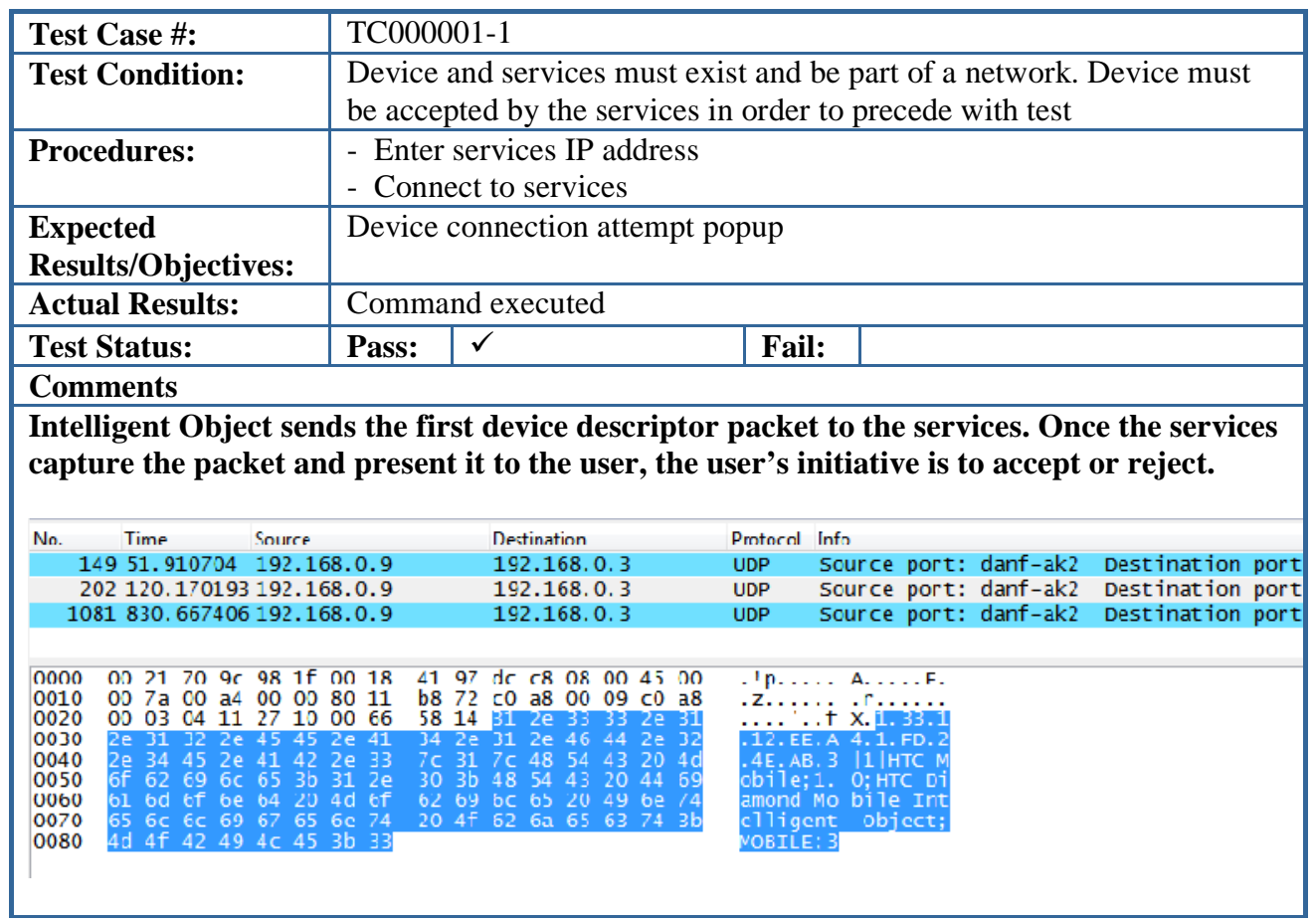

Table 2. Function Exchange 
International Journal of Software Engineering \& Applications (IJSEA), Vol.4, No.6, November 2013

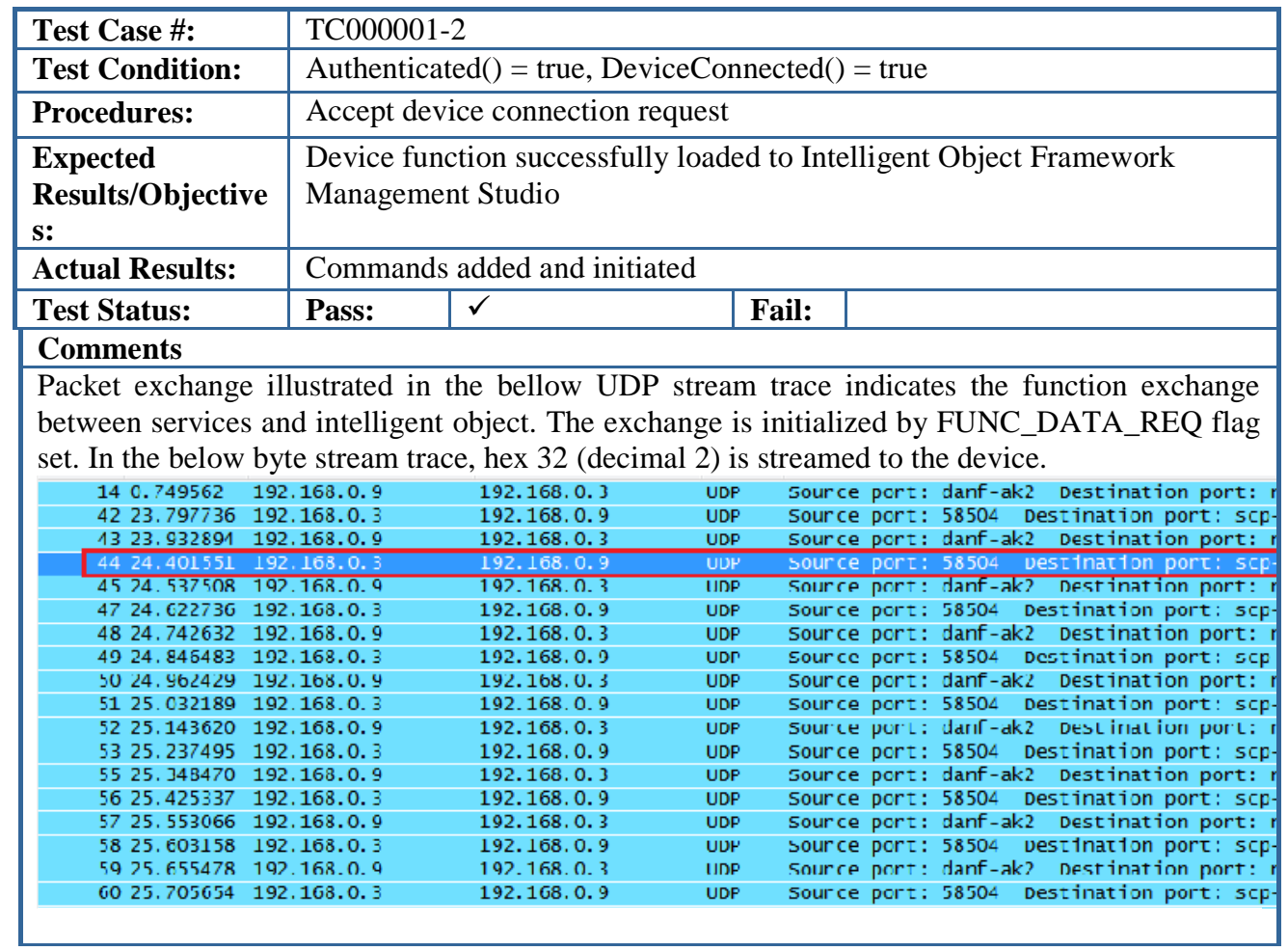


International Journal of Software Engineering \& Applications (IJSEA), Vol.4, No.6, November 2013

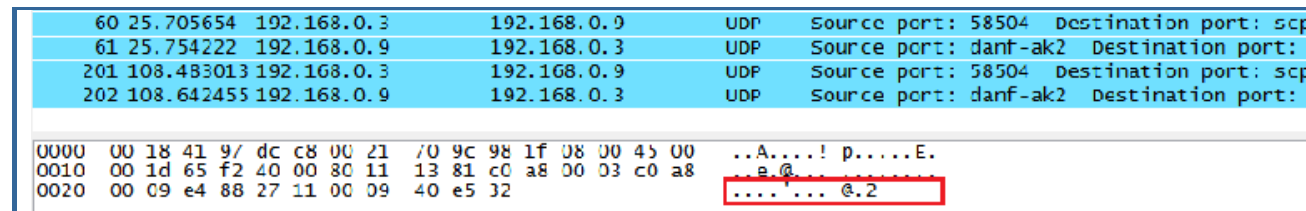

The intelligent object device responds by sending the first function to the services

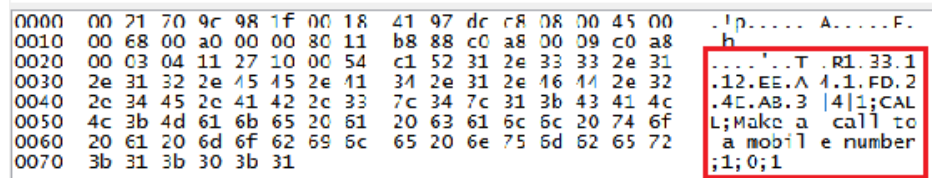

The services respond with an acknowledgement and request for parameters for the corresponding function

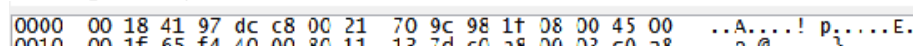

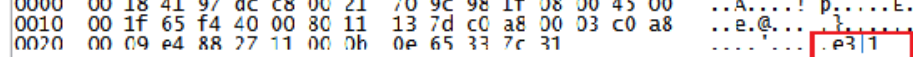

1

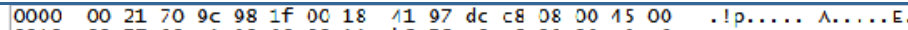

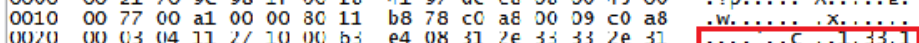

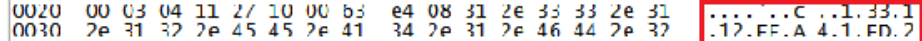

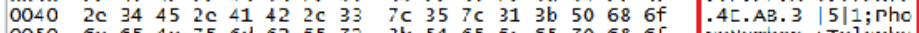

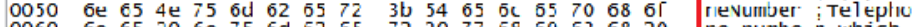

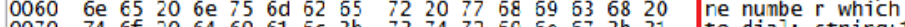

$\begin{array}{llllllllllllllllll}0070 & 74 & 6 f & 20 & 64 & 69 & 61 & 6 c & 3 b & 73 & 74 & 72 & 69 & 6 e & 6 ; & 3 b & 31 & \text { to dial; string; } 1 \\ 0080 & 32 & 32 & 33 & 3 b & 30\end{array}$

The process is finally completed once all function and parameter data has been exchanged with the device. The final status flag FIN is set and the device is added to the device collection.

$0000 \quad 0021709$ c 98 if 00184197 dc c8 $08004500 \quad$.!p.............

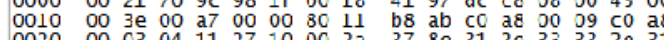

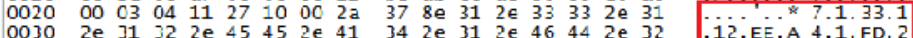

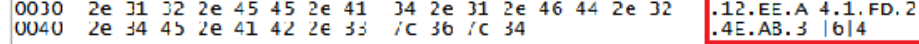

The stated byte trace correlates to the below graph by depicting transmission of each function and related parameters.

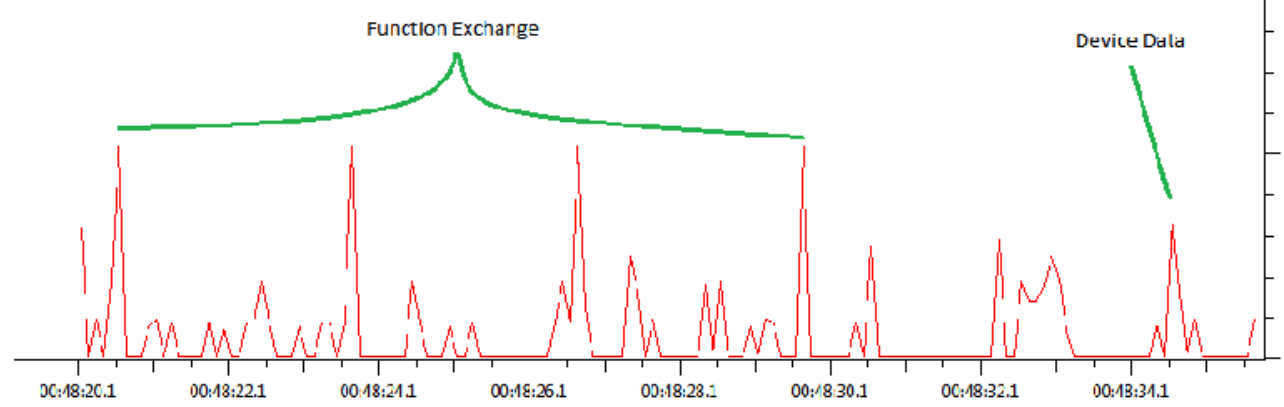

This proves the test case of function exchange

Table 3. Function Execution 
International Journal of Software Engineering \& Applications (IJSEA), Vol.4, No.6, November 2013

\begin{tabular}{|l|l|}
\hline Test Case \#: & TC000001-3 \\
\hline Test Condition: & LoggedOn( $)=$ true, DeviceActive ()$=$ true, PhoneNumber = 9769xxxx \\
\hline Procedures: & - Navigate to Functions \\
& - Select the appropriate command to execute \\
& - Define function parameters (if any) \\
\hline Expected & - Click on the Execute button \\
Results/Objective & Device response with a status of successful command execution \\
s: & Status expected: IOF_STS_0001 - COMMAND OK \\
\hline
\end{tabular}

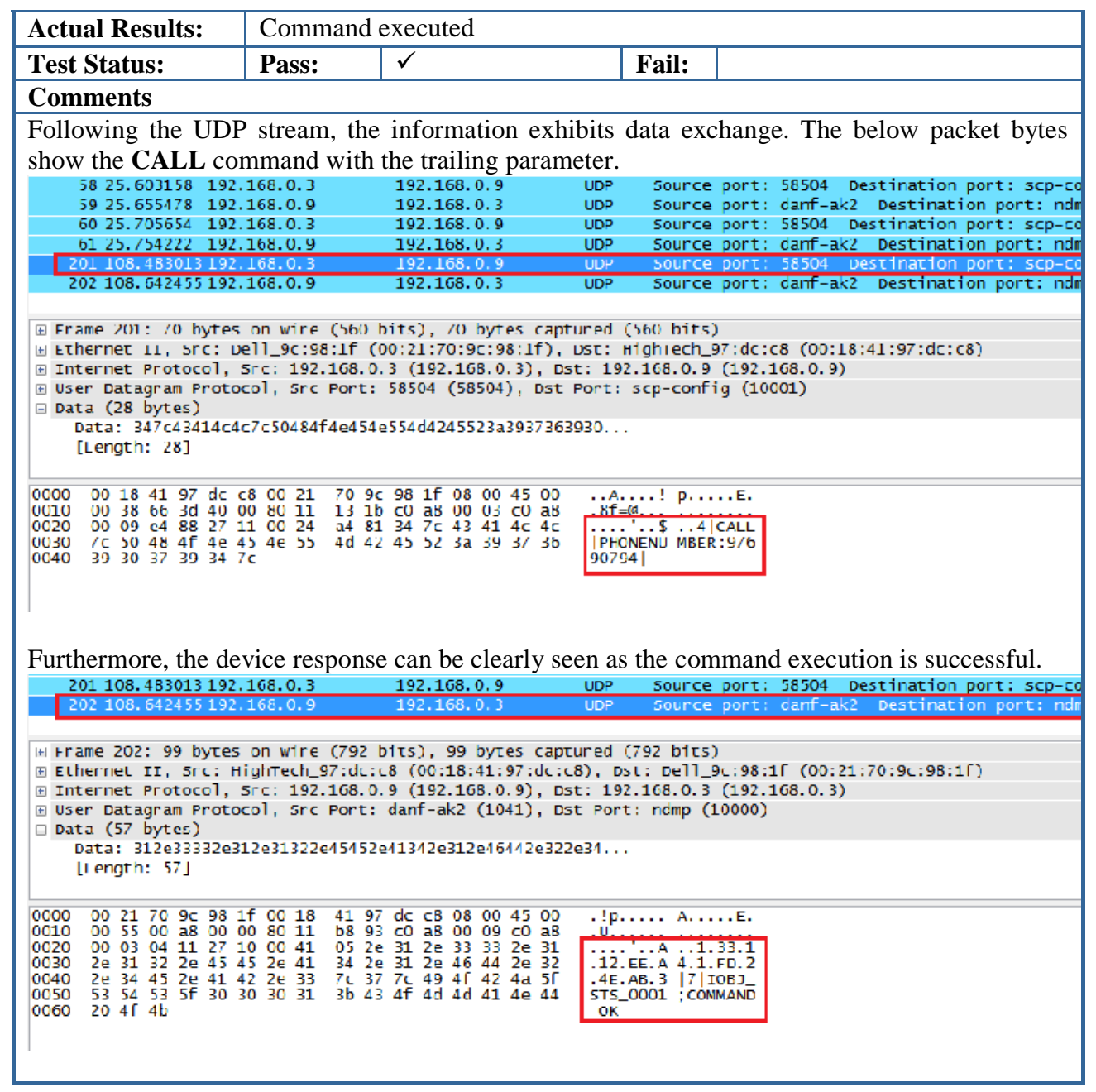

\section{DEPLOYMENT}

The deployment of Intelligent Object Framework with the additional HTC Mobile (Intelligent Object) and Tibbo Embedded Device (Intelligent Object 2) proved to be a success. The integration of Intelligent Object Framework consists of two primary steps in allowing full communication between the intelligent object and the intelligent object framework services, and integration of internal IOF components. First step states that the devices and the framework must be part of a network. The network endpoints, being the devices and the framework, must be able to communicate with each other. The second step that is required to be completed is the installation 
of Microsoft's SQL Server. As discussed earlier, the database keeps intelligent object data, thus the data is being retrieved on demand from the user. Application components are integrated at the beginning of the design of the internal framework infrastructure. The deployment of Intelligent Object Framework and the associated components can be summarized in Figure 4.

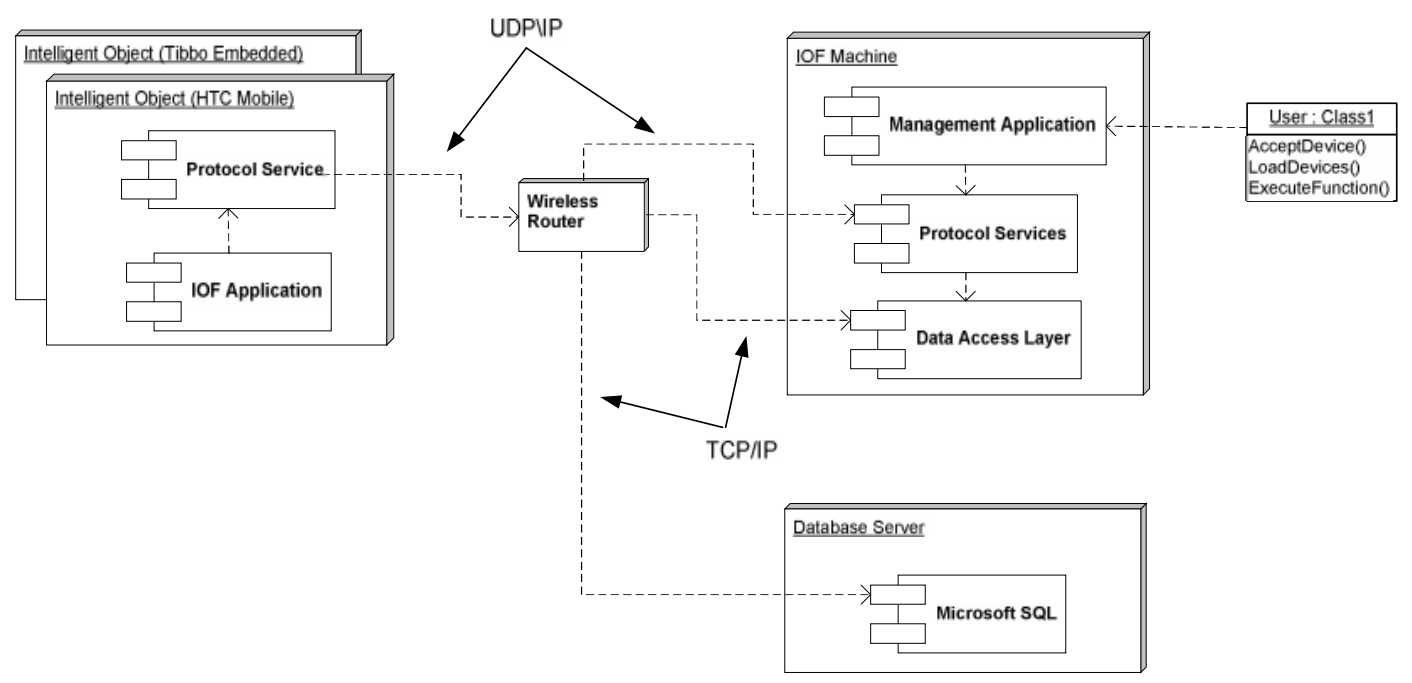

Figure4.IOF Deployment Diagram

\section{Conclusions}

It can be concluded that device control and management have become an essential functional addition to many devices ranging from remotely operated apparatuses, industrial equipment and network related devices. The ability to monitor, assess, fault rectify and manage devices wirelessly or over the Ethernet proves to be a fast, effective and cost reductive process, as companies and individuals no longer need to put up with resource constraints and budget for offsite support. The down side of having an infrastructure of different devices is managing and controlling individual components by using a range of different applications through different communication protocols. Each vendor has a set of protocols that applications need to conform to in order to satisfy production requirements. This also adds to the complexity of having to apply multiple firewall rules to allow application and device communication, increasing the threat to security of the internal network.

Whilst still in the primitive stage of development, the IOF shows another way of device control and management over the Ethernet or wireless medium, by implementation of the framework and its set of components which provide a bilateral communication protocol for multiple platform independent device monitoring and control. The primary requirement, for a successful completion of the proposed project, states that the IOF application must provide an interface for controlling two or more devices of different platforms.

\section{ACKNOWLEDGEMENTS}

Sasa Savic would like to thank Victoria University for offering the Research Training Scheme (RTS) for his PhD study. 
International Journal of Software Engineering \& Applications (IJSEA), Vol.4, No.6, November 2013

\section{REFERENCES}

[1] Massie W. W. and Underhill C.R. (1908). "The Future of the Wireless Art", Wireless Telegraphy \& Telephony: pp 67-71.

[2] Nikola Tesla (2007) "The Nikola Tesla Treasury”, Wilder Publications, 1st Edition, 8 August 2007.

[3] Bellis, M. "An outstanding scientist, Nikola Tesla paved the way for modern technology", http://inventors.about.com/od/tstartinventions/a/Nikola_Tesla.htm , viewed on 10 November 2013.

[4] Kahn R. E. and Cerf, V. G. (1999) "What Is The Internet (And What Makes It Work)", http://www.cnri.reston.va.us/what_is_internet.html, viewed on 10 November 2013.

[5] Savic S. and Shi, H. (2011), "An Intelligent Object Framework for Smart Living", Procedia Computer Science 5 (2011) 386-393.

[6] Savic, S. (2010) " Intelligent Object Framework", thesis for Master of Science in Computer Science, School of Engineering and Science, Victoria University, June 2010, 138 pages.

[7] Savic S. and Shi, H. (2013), "Detailed Design of Intelligent Object Framework", submitted toInternational Journal of Computer Networks \& Communications (IJCNC).

[8] Holburn, D. (1997) "Embedding the PC", viewed on 10 November 2013, http://www2.eng.cam.ac.uk/ dmh/chiprack/chiprack.html\#Embedded\%20Computer ,

[9] Barr, M. and Massa, A. (2006). Programming Embedded Systems with C and GNU Development Tools, 2nd edition, O'Reilly Media Inc.

[10] Embedded (2008). "Intel Microprocessor", http://www.embedded.com, viewed on 10 November 2013.

[11] Embedded (2010) "Embedded Timeline", http://www.embedded.com/timeline, retrieved on 2 May 2010.

[12] Al-Adaway, I. H. (2004) "Moral Technology" 6(3).

[13] Texas Instruments (2010). "OMAP 4 Mobile Applications Platform.", http://focus.ti.com//general/docs/wtbu/wtbuproductcontent.tsp?templateId=6123\&navigationId=1284 $3 \&$ contentId=53243\#chipDiagram, viewed on 10 November 2013.

[14] Embeded-System.Net (2009). "Open Multimedia Application Platform.", http://embeddedsystem.net/ti-omap-4-open-multimedia-application-platform-for-mobile-applications.html, viewed on 10 November 2013.

[15] Bluetooth (2010). "Overview of Bluetooth Operation",retrieved on 2 May 2010, http://www.bluetooth.com/English/Technology/Works/Pages/Overview_of_Operation.aspx .

[16] Sriskanthan N., Tan, F. and Karande,A. (2002). "Bluetooth based home automation system." Microprocessors and Microsystems 26(6): 281-289.

\section{Authors}

Sasa Savicis a part-time $\mathrm{PhD}$ student at Victoria University in Melbourne, Australia. He obtained Advanced Diploma of Computer Systems Engineering in 2002 from Royal Melbourne Institute of Technology (RMIT), Australia. He received Bachelor of Science and Master of Science in Computer Science from Victoria University in 2006and 2010, respectively. He is currently working at Telstra, the largest Australian telecommunications company, as a senior software engineer.

Dr. Hao Shi is an Associate Professor in College of Engineering and Science, Victoria University, Melbourne, Australia. She completed her Bachelor of Engineering degree at Shanghai Jiao Tong University, China and obtained her PhD in the area of Computer Engineering at University of Wollongong. She has been actively engaged in R\&D and external consultancy activities. Her research interests include p2p Network, LocationBased Services, Web Services, Robotics Vision, Visual Communications, Internet and

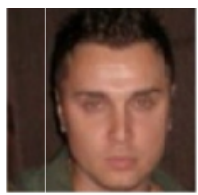
Wireless Technologies.

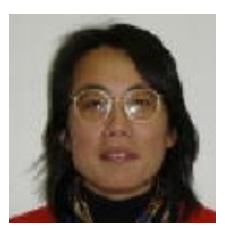

